\title{
ONE AXIOMATIC SYSTEM FOR THE ONTOLOGY REVISION*
}

\author{
Yu Sun, ${ }^{1,2}$ Yuefei Sui, ${ }^{2}$ and Zhiping $\mathrm{Li}^{3}$ \\ ${ }^{1}$ School of Computer Science and Information Technology \\ Yunnan Normal University, Kunming 650092 \\ sunyu_km@hotmail.com \\ ${ }^{2}$ Key Laboratory of Intelligent Information Processing \\ Institute of Computing Technology \\ Chinese Academy of Sciences, Beijing 100080 \\ yfsui@ict.ac.cn \\ ${ }^{3}$ Education Technology Center \\ Yunnan Normal University, Kumming 650092 \\ ynnulzp@sohu.com
}

\begin{abstract}
An ontology consists of concepts and the subsumption relation between these concepts, and is assumed to be a tree under the subsumption retation. In the process of building and maintaining ontologies, new statements which may contradict with exiting statements are added to the ontologies constantly. The ontology revision is necessary to accommodate new statements. In terms of the method of the axiomatization, one axiom system for the ontology revision, called the $Z$ axiom system, is given, which is proved to satisfy the principles of the success, consistency and minimal change. Unlike the belief revision which is monotonic, the ontology revision may not be monotonic, and not only extracts some statements contradictory with a revising statement, extracts statements which are not contradictory with the revising statement, but also adds new statements to keep the tree structure of the revised ontology and satisfy the minimal change. One concrete ontology revision operator is proposed, which is proved to satisfy the $Z$ axiom system.
\end{abstract}

Keywords: Ontologies, Belief revision, Subsumption relation, Default inheritance

* This work is supported by the NSF (grant no. 60273019,60496326, 60573063, and 60573064), the National 973 Programme (grants no. 2003CB317008 and G1999032701) and the Foundation of Yumnan Province (grants no. 04F00062, 2004YX42, 2004F0017Q, 03Y312D and 2005F0022Q).

Please use the following format when citing this chapter:

Sun, Y., Sui, Y., Li, Z, 2006, in IFIP International Federation for Information Processing, Volume 228, Intelligent Information Processing III, eds. Z. Shi, Shimohara K., Feng D., (Boston: Springer), pp. 91-100. 


\section{Introduction}

A general approach for studying belief revision is to provide a set of postulates for belief revision functions. These postulates constrain what revision functions should satisfy in the process of revision, but say little about how to implement these functions. The AGM approach $[2,3]$ perhaps provides the best-known set of such postulates and an extended discussion on the postulates was given in $[4,5]$. The AGM axiom system is not very appropriate for the iterated belief revision [1]. Hence, Darwiche and Pearl [1] put forward a wellknown proposal which extends the AGM axiom system with four additional postulates for the iterated belief revision $[6,7]$.

The belief revision has three basic principles $[8,9]$ : the principles of the success, the consistency and the minimal change. Furthermore, the belief revision is monotonic. That is, given two knowledge base $K$ and $K^{\prime}$, if $K \vdash K^{\prime}$, then $K \circ \alpha \vdash K^{\prime} \circ \alpha$, where $K \circ \alpha$ is the knowledge base resulted from revising $K$ by $\alpha$. For a knowledge base $K$ and a revising statement $\alpha$, a belief revision is not to revise statements in $K$, but to extract some statements in $K$ to make $K^{\prime} \cup\{\alpha\}$ consistent for the remaining subset $K^{\prime}$ of $K$.

McGuinness [10] proposed that a simple ontology should contain the following items:

(1) finite controlled (extensible) vocabulary;

(2) unambiguous interpretation of classes and term relationships;

(3) strict hierarchical subclass relationships between classes.

In [11], the authors classified the currently used ontology languages according to whether ontologies contain concepts, taxonomies, relations and functions, axioms and instances. For the simplicity of discussion, we assume that an ontology consists of the following three kinds of statements and their negations:

- the subsumption relation between concepts: $C \sqsubseteq D$;

- a concept having a property: $C \Rightarrow \varphi$;

- a concept defaultly having a property: $C \Rightarrow_{d} \varphi$,

where $C, D$ are concepts, $\varphi, \psi$ are properties. We assume that $O$ is a tree under the subsumption relation between concepts.

The ontology revision is a process of changing ontologies to accommodate statements that are possibly inconsistent with existing statements. For an ontology $O$ and a revising statement $\theta$, let $O \circ \theta$ be the ontology that results from revising $O$ by $\theta$. The ontology revision has the following properties which the belief revision does not have:

$\diamond$ The ontology revision is not monotonic. That is, for any ontologies $O, O^{\prime}$ and a revising statement $\theta$, if $O \vdash O^{\prime}$ then it is possible that $O \circ \theta \forall \forall O^{\prime} \circ \theta$.

$\diamond$ To keep the tree structure of $O \circ \theta$, according to the structure of $O$, we not only extract a set of statements $S$ from $O$ to ensure that $(O \cup\{\theta\})-S$ 
is consistent, but extract another set of statements $\Delta$ other than $S$ from $O$ and add a set of new statements $T$ other than $\{\theta\}$ to $O \circ \theta$.

By the axiomatization, an axiom system for the ontology revision, called the $Z$ axiom system, will be proposed, and proved to satisfy the principles of success, consistency and minimal change. According to the inconsistency of $\theta$ with $O, T h(O)$ and $O^{\mathrm{CWA}}$, a concrete ontology revision operator will be given and proved to satisfy the $Z$ axiom system.

The paper is organized as follows: in section 2 , the definition and presuppositions of ontologies are given, and the logical implications in ontologies are discussed; in section 3, we give the presuppositions for the ontology revision and according to the structure of ontologies, we propose an axiom system, called the $Z$ axiom system, for the ontology revision satisfying the principles of success, consistency and minimal change. In section 4 , a concrete ontology revision operator 0 is given, which is proved to satisfy the $Z$ axiom system. The last section concludes the paper.

\section{Ontologies}

In this section, we firstly give the definition and presuppositions of ontologies, then discuss the logical implications in ontologies.

Definition 1. An antology $O$ consists of

- a set of concepts and properties;

- four binary relations: the subsumption relation $\sqsubseteq$ between concepts; the inheritance relation $\Rightarrow$ between concepts and properties, the default inheritance relation $\Rightarrow d$ between concepts and properties; and

- a set of positive statements of forms $C \leq D|C \Rightarrow \varphi| C \Rightarrow d \varphi$ and their negations of forms $C \notin D|C \nRightarrow \varphi| C \nRightarrow d \varphi$, where $C, D$ are concepts and $\varphi, \psi$ are properties.

We use $U$ to denote the set of concepts and properties in $O$. For any concept $C$, concept $C^{*}$ is a $\sqsubseteq$-minimal super-concept of $C$ such that there is no $D$ such that $C \sqsubseteq D \in O$ and $D \equiv C^{*} \in O$.

For the simplicity, we assume that $O$ is a tree under subsumption relation $E$, i.e., for any concept $C, C^{*}$ is unique. This guarantees that as a default theory, $O$ under the implication rules has a unique extension.

Given an ontology $O$, concepts $C, D$ and properties $\varphi$ and $\psi$ in $U, O$ is a default theory with defaults. The reasoning in $O$ is the reasoning of default theory $(O, W)$, where $W$ is the set of defaults and implication rules showed in 
the following:

$$
\begin{array}{ll}
\text { the transitivity of } \sqsubseteq & \frac{C \sqsubseteq D, D \subseteq E}{C \sqsubseteq E} ; \\
\text { the inheritance rule } & \frac{C \sqsubseteq D, D \Rightarrow \varphi}{C \Rightarrow \varphi} ; \\
\text { the default inheritance rule } & \frac{C \sqsubseteq D, D \Rightarrow_{d} \varphi: C \Rightarrow_{d} \varphi}{C \Rightarrow_{d} \varphi} .
\end{array}
$$

By the presupposition that $O$ is a tree under $\sqsubseteq$, as a default theory, $O$ has a unique extension. Let $T h(O)$ be the unique extension of $O$. Define $\vdash$ to be the implication relation defined by $T h(O)$, i.e., for any statement $\delta$,

$$
O \vdash \delta \text { iff } \delta \in T h(O)
$$

The default theory of $T h(O)$ under the closed world assumption has a unique extension, denoted by $O^{\mathrm{CWA}}$, and

$$
O^{\mathrm{CWA}}=\{\neg \delta: O \forall \delta\} \cup \operatorname{Th}(O),
$$

where $\delta$ is a positive statement.

Definition 2. An ontology $O$ is inconsistent if there is a statement $\delta$ such that $\delta, \neg \delta \in O^{\mathrm{CWA}}$.

Remark. Similar to belief revision, there are two kinds of ontology revision: ontology-set revision and ontology-base revision. In this paper, an ontology $O$ is an ontology base, and its ontology set is $T h(O)$.

\section{The ontology revision}

In this section, we shall give firstly an example of the ontology revision, and secondly the presuppositions, and then the axioms for the ontology revision.

\subsection{One example}

Let us take a look at the following example. Example 1 shows our intuition for the ontology revision.

Example 1. We believe that sparrow and penguin are two kinds of bird and bird can fly. Formally, the ontology can be represented by

$$
O=\{\text { sparrow } \sqsubseteq \text { bird, penguin } \sqsubseteq \text { bird, bird } \Rightarrow \text { flying }\} .
$$

Assume that later, we find that penguin actually cannot fly, that is,

$$
\theta=\text { penguin } \not \text { flying. }
$$

Then we do not believe that bird can fly, since penguin is a bird. In this example, $O \cup\{\theta\}$ is inconsistent and $\neg \theta \in T h(O)$, since $\neg \theta=$ penguin $\Rightarrow$ 
flying can be inferred from $O$ by the inheritance. Formally,

$$
\begin{aligned}
& O \circ \theta=\text { sparrow } \sqsubseteq \text { bird, penguin } \sqsubseteq \text { bird, } \\
& \text { penguin } \nRightarrow \text { flying } \\
& S=\{\text { bird } \Rightarrow \text { flying }\} \\
& \Delta=\emptyset \text {; } \\
& T=\emptyset \text {. }
\end{aligned}
$$

This example can be realized by case 2.4 .2 in section 4 . The following figure shows $O$ and $O \circ \theta$.
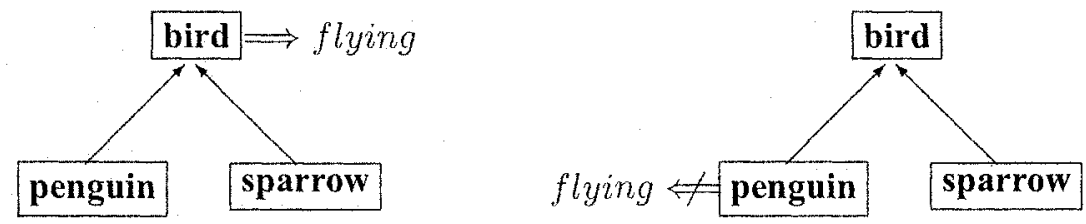

Fig. 1. $O$ and $O \circ \theta$

Remark. In example 1, intuitively, we shall still believe that sparrow can fly after revision, since sparrow and penguin are two different kinds of bird, and the change of property penguin being ftying should not affect that of sparrow. In other words, although sparrow $\Rightarrow$ flying is not stated explicitly in $O$, it can be inferred from $O$ by the inheritance and this kind of implicit statements is what $O$ has inherently. For the simplicity, we do not consider the preservation of such implicit statements in the ontology revision.

\subsection{The presuppositions for the ontology revision}

For an ontology $O$ to be revised and a revising statement $\theta$, we have the following presuppositions:

1. $O$ is consistent;

2. $O \circ \theta$ is an ontology;

3. $\theta$ is of forms: $C \sqsubseteq D, C \unrhd D, C \Rightarrow \varphi$ and $C \not \varphi$;

4. The ontology revision satisfies the principle of the success: $\theta \in O \circ \theta$; the principle of the consistency: $O \circ \theta$ is consistent if $O$ is consistent; and the principle of the minimal change: the symmetric difference between the set of statements in $O$ and in $O \circ \theta$, denoted by $\operatorname{sd}(O, O \circ \theta)$, is minimal, that is, let $\Gamma(O)$ be the set of statements in $O$, then $|(\Gamma(O)-\Gamma(O \circ \theta)) \cup(\Gamma(O \circ \theta)-\Gamma(O))|$ is minimal.

5. For the iterated ontology revision, the revising statements are always consistent with each other. For example, if $O \circ \varphi$ is an ontology to be revised and $\psi$ is a revising statement then $\varphi$ is consistent with $\psi$. 
6. In order to keep the tree structure of $O \circ \theta$, we may add to $O \circ \theta$ new statements which are consistent with $O \cup\{\theta\}$ after the extraction of the statement of the subsumption relation between two concepts $C$ and $D$ if $\theta=C \square D$ and $O \cup\{\theta\}$ is inconsistent; and a set of statements may be extracted from $O$ if $\theta=C \sqsubseteq D$ and $O \cup\{\theta\}$ is inconsistent.

Let $S$ be the smallest set of statements extracted from $O$ to ensure $(O \cup$ $\{\theta\})-S$ is consistent; $\Delta$ the smallest set of statements extracted from $O$ other than $S$; and $T$ the smallest set of new statements $\notin O \cup\{\theta\}$ added to $O \circ \theta$. To keep the tree structure of the revised ontology, we assume that

$$
O \circ \theta=((O \cup\{\theta\})-S-\Delta) \cup T,
$$

where $S \cap \Delta=\emptyset$.

\subsection{The axioms for the ontology revision}

To give the axioms for the ontology revision, we firstly notice the difference between the ontology revision and belief revision. In the belief revision, to be revised is a knowledge base $K$ and to revise is a formula $\alpha$. Every statement is constructed from atomic formulas in terms of the logical connectives. In the ontology revision, to be revised is an ontology $O$ and to revise is a statement $\theta$ which is atomic.

Based on the discussion in section 3.1 and 3.2, we propose the $Z$ axiom system for the ontology revision:

$Z 0 . O \circ \theta$ is an ontology.

$\mathrm{Z} 1 . O \circ \theta$ is consistent if $\theta$ is not contradictory.

Z2. $\theta \in O \circ \theta$.

Z3. If $O \cup\{\theta\}$ is consistent, then $O \circ \theta=(O \cup\{\theta\})-\Delta$.

Z4. If $O \cup\{\theta\}$ is inconsistent, then $O \circ \theta=((O \cup\{\theta\})-S-\Delta) \cup T$.

Z5. If $O \circ \theta+\delta$ then $(O \circ \delta) \circ \theta \equiv O \circ \theta$.

The $Z$ axiom system is a combination of the AGM axiom and the DP axiom in some sense, except that if $K \cup\{\alpha\}$ is consistent then $K \circ \alpha \equiv K \cup\{\alpha\}$. By Z3, even though $O \cup\{\theta\}$ is consistent, something has to be extracted from $O$ to make $O$ satisfy the presuppositions on $O$. For the ontology revision, such a combination is appropriate, because of $\delta$ being atomic.

Theorem 1. The Z axiom system satisfies the principles of success, consistency and minimal change.

Proof. By $Z 2$, the $Z$ axiom system satisfies the principle of success. By $Z 0$ and $\mathrm{Zl}$, the $\mathrm{Z}$ axiom system satisfies the principle of consistency.

If $O \cup\{\theta\}$ is inconsistent, then by $Z 4, O \circ \theta=((O \cup\{\theta\})-S-\Delta) \cup T$. By presupposition 4 and the definitions of $\Delta, S$ and $T, s d(O, O \circ \theta)=|S \cup \Delta \cup T|$ is minimal. Similarly, we can prove that $\operatorname{sd}(O, O \circ \theta)$ is minimal when $O \cup\{\theta\}$ is consistent. 
Hence, the $Z$ axiom system satisfies the principle of minimal change.

Remark. Here, the principle of the minimal change is syntactical. The minimal change in the belief revision has three readings: syntactical, semantical (i.e., the minimal distance of models), and set-theoretic (taking knowledge bases as sets).

\section{One concrete ontology revision o}

In this section, we define an ontology revision operator $\circ$ which satisfies the $\mathrm{Z}$ axiom system.

Given an ontology $O$ and a revising statement $\theta$, assume that $\theta$ is not contradictory (otherwise, let $O \circ \theta=\emptyset$ ). By presupposition 3, $\theta$ is of one of the following forms:

$$
C \sqsubseteq D ; C \nsubseteq D ; C \Rightarrow \varphi ; C \nRightarrow \varphi,
$$

and $O \cup\{\theta\}$ may be consistent or not.

When $O \cup\{\theta\}$ is consistent, if $\theta$ is positive then $\theta \in O, \theta \in T h(O)$ or $\neg \theta \in O^{\mathrm{CWA}}$; otherwise, $\theta \in O, \theta \in T h(O)$ or $\theta \in O^{\mathrm{CWA}}$.

When $O \cup\{\theta\}$ is inconsistent, $\neg \theta \in O$ or $\neg \theta \in T h(O)$.

To discuss the consistence of $O \cup\{\theta\}$, the introduction of $O^{\mathrm{CWA}}$ is necessary. For example, if $O=\{C \sqsubseteq E, D \sqsubseteq E\}$ and $\theta=C \sqsubseteq D$, then $\theta \notin O, \theta \notin T h(O)$ and $\neg \theta \notin O, \neg \theta \notin T h(O)$, but $\neg \theta \in O^{\mathrm{CWA}}$.

We give a concrete ontology revision operator $\circ$, based on the consistence of $O \cup\{\theta\}$ and the forms of $\theta$.

Case 1. $O \cup\{\theta\}$ is consistent.

Case 1.1. $\theta=C \nsubseteq D$ or $C \neq \varphi$.

$\begin{array}{ll}\text { Case 1.1.1. } \theta \in O . & \text { Let } O \circ \theta=O . \\ \text { Case 1.1.2. } \theta \in T h(O) . & \text { Let } O \circ \theta=O \cup\{\theta\} . \\ \text { Case 1.1.3. } \theta \in O^{\text {CWA }} . & \text { Let } O \circ \theta=O \cup\{\theta\} .\end{array}$

Case 1.2. $\theta=C \sqsubseteq D$.

Case 1.2.1. $\theta \in O$. Let $O \circ \theta=O$.

Case 1.2.2. $\theta \in T h(O)$. Let $O \circ \theta=O \cup\{\theta\}$.

Case 1.2.3, $\neg \theta \in O^{\mathrm{CWA}}-T h(O)$.

Let $\Delta=\{C \sqsubseteq E \in O: D \sqsubseteq E \notin T h(O)\}$.

Then, $O \circ \theta=(O \cup\{\theta\})-\Delta$.

Case 1.3. $\theta=C \Rightarrow \varphi$.

Case 1.3.1. $\theta \in O$. Let $O \circ \theta=0$.

Case 1.3.2. $\theta \in T h(O)$. Let $O \circ \theta=O \cup\{\theta\}$.

Case 1.3.3. $\neg \theta \in O^{\mathrm{CWA}}-T h(O)$. Let $O \circ \theta=O \cup\{\theta\}$.

Case 2. $O \cup\{\theta\}$ is inconsistent.

Assume that $O \circ \theta=((O \cup\{\theta\})-S-\Delta) \cup T$. 
Case 2.1. $\theta=C \not D D$.

Case 2.1.1. $C \subseteq D \in O$.

Let $S=\{C \subseteq D\} ; \Delta=\emptyset$; and $T=\left\{C \sqsubseteq D^{*}\right\}$.

Case 2.1.2. $C \sqsubseteq D \in T h(O)$.

Let

$$
\begin{aligned}
& S=\{C \sqsubseteq E \in O: E \sqsubseteq D \in T h(O)\} \\
& \Delta=\emptyset ; T=\left\{C \sqsubseteq D^{*}\right\} .
\end{aligned}
$$

Case 2.2. $\theta=C \sqsubseteq D$.

Case 2.2.1. $C Z D \in O$.

Let

$$
\begin{aligned}
& S=\{C \nsubseteq D\} \\
& \Delta=\{C \subseteq E \in O: D \subseteq E \notin T h(O)\} ; \\
& T=\emptyset .
\end{aligned}
$$

Case 2.2.2. $C \square D \in T h(O)$.

Let

$$
\begin{aligned}
S= & \{C \not \varphi \in O: D \Rightarrow \varphi \in T h(O)\} \cup \\
& \{C \nsubseteq E \in O: D \sqsubseteq E \in T h(O)\} ; \\
\Delta= & \{C \sqsubseteq E \in O: D \sqsubseteq E \notin T h(O)\} ; \\
T=\emptyset . &
\end{aligned}
$$

Case 2.3. $\theta=C \Rightarrow \varphi$.

Case 2.3.1. $C \nRightarrow \varphi \in O$.

Let $S=\{C \not \varphi\}, \Delta=T=\emptyset$.

Case 2.3.2. $C \not p \in T h(O)$.

Let

$$
\begin{aligned}
& S=\{E \nRightarrow \varphi \in O: E \subseteq C \in T h(O)\} \\
& \Delta=T=\emptyset .
\end{aligned}
$$

Case 2.4. $\theta=C \not \varphi$.

Case 2.4.1. $C \Rightarrow \varphi \in O$.

Let $S=\{C \Rightarrow \varphi\}, \Delta=T=\emptyset$.

Case 2.4.2. $C \Rightarrow \varphi \in T h(O)$.

Let

$$
\begin{aligned}
& S=\{D \Rightarrow \varphi \in O: C \sqsubseteq D \in T h(O)\} \\
& \Delta=T=\emptyset .
\end{aligned}
$$

By the definition of $\circ$, we have the following theorem.

Theorem 2. o satisfies the $Z$ axiom system.

Proof. It is a routine to verify that $\circ$ satisfies the $Z$ axiom system. We prove the theorem for case 2.1.1.

In case 2.1.1, $\theta=C \nsubseteq D, C \sqsubseteq D \in O$ and $O \cup\{\theta\}$ is inconsistent.

Let $S=\{C \sqsubseteq D\} ; \Delta=\emptyset$; and $T=\left\{C \sqsubseteq D^{*}\right\}$, then

$$
O \circ \theta=((O \cup\{\theta\})-S-\Delta) \cup T .
$$

By the definitions of $S, \Delta, T$ and $O \circ \theta$, we can see that $C$ has only one E-least super-concept $D^{*}$ in $O \circ \theta ; T$ is consistent with $(O \cup\{\theta\})-S-\Delta$ 
and $O \circ \theta$ is consistent. Hence, $\mathrm{Z} 0$ and $\mathrm{Zl}$ are satisfied. $\mathrm{Z} 2$ is satisfied since $\theta \notin S \cup \triangle ; Z 3$ is satisfied, since $\theta, \neg \theta \in O \cup\{\theta\}$ and $O \cup\{\theta\}$ is inconsistent.

By the definitions of $O \circ \theta, S, \Delta$ and $T$, it can be proved that $S$ is the smallest set of statements extracted from $O$ to ensure $(O \cup\{\theta\})-S$ is consistent; and $\Delta$ is the smallest set of statements extracted from $O$ and $T$ is the smallest set of statements added to $O \circ \theta$ to do the reclassification of concepts and to keep the tree structure of $O \circ \theta$ as what has been discussed in section 3.2. Hence, $\mathrm{Z} 4$ is satisfied.

For Z5, we only consider the case that $\delta=C \sqsubseteq D^{*}$, and other cases are similar. By our assumption, $C \sqsubseteq D \in O$, then $O \cup\{\delta\}$ is consistent. By case 1.2.3, we have

$$
O \circ \delta=(O \cup\{\delta\})-\{C \sqsubseteq D\} .
$$

Hence, $C \sqsubseteq D \notin O \circ \delta$ and $(O \circ \delta) \cup\{\theta\}$ is consistent. By case 1.1.3, we have

$$
O \circ \delta \circ \theta=O \circ \delta \cup\{\theta\}=O \circ \theta \text {. }
$$

\section{Conclusion and further works}

In terms of the method of axiomatization, an axiom system, called the $Z$ axiom system, for the ontology revision is given, which is proved to satisfy the principles of the success, consistency and minimal change. The ontology revision satisfying the $Z$ axiom system has the following properties:

(1) if $\theta$ is consistent with $O$, then $O \circ \theta=(O \cup\{\theta\})-\Delta$;

(2) otherwise, $O \circ \theta=((O \cup\{\theta\})-S-\Delta) \cup T$,

where $S, \Delta$ and $T$ are sets of statements. The $Z$ axiom system contains the axioms for the iterated revision:

(3) if $O \circ \theta+\delta$ then $(O \circ \delta) \circ \theta \equiv O \circ \theta$.

A concrete ontology revision is given, which is based on the cases that $O \cup\{\theta\}$ is consistent or not.

(4) If $\theta$ is consistent with $O$, then $\theta \in O, \theta \in T h(O), \theta \in O^{\mathrm{CWA}}$ if $\theta$ is negative or $\neg \theta \in O^{\mathrm{CWA}}$ if $\theta$ is positive.

(5) otherwise, $\neg \theta \in O$ or $\neg \theta \in T h(O)$.

Then, a concrete ontology revision function is given, which is proved to satisfy the $\mathrm{Z}$ axiom system.

In discussing the properties of natural kind concepts, the induction is necessary. For example, if every instance of bird we have found has feathers, then we conclude that bird has feathers by induction. Our next work will include the induction process in the ontology revision; and the logical properties between $C \Rightarrow \varphi$ and $C \Rightarrow_{d} \varphi$; the structure of concepts and properties; and the structure of statements in ontologies.

\section{References}


[1] A. Darwiche and J. Pearl. On the logic of iterated belief revision. Artificial Intelligence, $89(1-2): 1-29,1997$

[2] C. E. Alchourrón, P. Gärdenfors and D. Makinson. On the logic of theory change: partial meet contraction and revision functions. Journal of Symbolic Logic, 50:510-530, 1985.

[3] P. Gärdenfors. Knowledge in Flux: Modelling the Dynamics of Epistemic States. The MIT Press, Cambridge, MA, 1988.

[4] S. O. Hansson. A Textbook of Belief Dynamics. Applied Logic Series. Kluwer Academic Publishers, 1999.

[5] H. Rott. Change, Choice and Inference - A Study of Belief Revision and Nonmonotonic Reasoning. Oxford: Clarendon Press, 2001.

[6] C. Boutilier and M. Goldszmidt. Revision by conditional beliefs. In Proceedings of the AAAI National Conference on Artificial Intelligence, pages 649-654. Morgan Kaufmann, Washington, D.C., July 1993.

[7] O. Papini. Iterated revision operations stemming from the history of an agent's observations. In M.-A. Williams and H. Rott, editors, Frontiers in Belief Revision, 22:279-301, Kluwer Academic Publishers, 2001.

[8] D. Dubois and H. Prade. Introduction: Revising, Updating and Combining knowledge. In D. M. Gabbay (eds.), Handbook of Defeasible Reasoning and Uncertainty Management Systems, 3:1-16, 1998.

[9] S. O. Hansson. Revision of Belief Sets and Belief Bases. In D. M. Gabbay (eds.), Handbook of Defeasible Reasoning and Uncertainty Management Systems, 3:17-75, 1998.

[10] D. L. McGuinness. Ontologies come of age. In D. Fensel, J. Hendler, H. Lieberman and W. Wahlster, (eds.), Spinning the Semantic Web: Bringing the World Wide Web to Its Full Potential. MIT Press, 2002.

[11] A. Gómez-Pérez and O. Corcho. Ontology languages for the semantic web. IEEE Intelligent Systems, 54-60, 2002.

[12] Y. Sin and Y. Sui. The Ontology Revision. In Proc. of International Joint Conference of Artificial Intelligence, 1583-1584, 2005.

[13] C. B. Cross. Nonmonotonic inconsistency. Artificial Intelligence, 149(2):161-178, 2003.

[14] R. Reiter, A Logic for Default Reasoning. Artificial Intelligence, 13:81-132, 1980. 